\title{
Risk factors for biliary tract and ampullary carcinomas and prophylactic surgery for these factors
}

\author{
Masaru Miyazaki ${ }^{1}$, Tadahiro Takada ${ }^{2}$, Shuichi Miyakawa ${ }^{3}$, Kazuhiro Tsukada $^{4}$, Masato Nagino $^{5}$, \\ Satoshi Kondo ${ }^{6}$, Junit Furuse ${ }^{7}$, Hiroya Saito ${ }^{8}$, Toshio Tsuyuguchi ${ }^{9}$, Kazuo Chijima ${ }^{10}$, Fumio Kimura ${ }^{1}$, \\ Hideyuki Yoshitomi ${ }^{1}$, Satoshi Nozawa ${ }^{1}$, Masahiro Yoshida ${ }^{2}$, Keita Wada ${ }^{2}$, Hodaka Amano ${ }^{2}$, \\ and Fuminiko MiURA ${ }^{2}$ \\ ${ }^{1}$ Department of General Surgery, Chiba University Graduate School of Medicine, 1-8-1 Inohana, Chuo-ku, Chiba 260-8670, Japan \\ ${ }^{2}$ Department of Surgery, Teikyo University School of Medicine, Tokyo, Japan \\ ${ }^{3}$ Department of Gastroenterological Surgery, Fujita Health University, Toyoake, Japan \\ ${ }^{4}$ Department of Surgery and Science, Graduate School of Medicine and Pharmaceutical Science for Research, University of Toyama, Toyama, \\ Japan \\ ${ }^{5}$ Division of Surgical Oncology, Department of Surgery, Nagoya University Graduate School of Medicine, Nagoya, Japan \\ ${ }^{6}$ Department of Surgical Oncology, Hokkaido University Graduate School of Medicine, Sapporo, Japan \\ ${ }^{7}$ Hepatobiliary and Pancreatic Oncology Division, National Cancer Center Hospital East, Chiba, Japan \\ ${ }^{8}$ Department of Radiology, Asahikawa Kosei General Hospital, Asahikawa, Japan \\ ${ }^{9}$ Department of Medicine and Clinical Oncology, Chiba University Graduate School of Medicine, Chiba, Japan \\ ${ }^{10}$ Department of Surgical Oncology and Regulation of Organ Function, Miyazaki University School of Medicine, Miyazaki, Japan
}

\begin{abstract}
Curative resection is the only treatment for biliary tract cancer that achieves long-term survival. However, patients with advanced biliary tract cancer have only a limited prognosis even after radical surgical resection. Thus, to improve the longterm results, the early detection of biliary tract cancer and subsequent cure seem to be essential. The purpose of this study was to review the literature concerning the risk factors for cancerous and precancerous lesions of the biliary tract, and prophylactic surgery for these factors. It has been reported that pancreaticobiliary maljunction (PBM) with bile duct dilatation is a risk factor for gallbladder cancer and bile duct cancer, while PBM without bile duct dilatation is a risk factor for gallbladder cancer. Thus, in the former group, a prophylactic excision of the common bile duct and gallbladder should be recommended, while in the later group, a prophylactic cholecystectomy without bile duct resection may be the appropriate surgical procedure. It has also been reported that primary sclerosing cholangitis (PSC) is a risk factor for cholangiocarcinoma. Patients with PSC often develop advanced cholangiocarcinoma with a poor prognosis. In patients with PSC, therefore, strict follow-up should be recommended. Adenoma and dysplasia have been regarded as precancerous lesions of gallbladder cancer. A polypoid lesion of the gallbladder that is sessile, has a diameter greater than $10 \mathrm{~mm}$, and /or grows rapidly, is highly likely to be cancerous and should be resected. Although gallstones seem to be closely associated with gallbladder cancer, there is no evidence of a direct causal relationship between gallstones and gallbladder cancer. Thus, a cholecystectomy is not advised for asymptomatic cholecystolithiasis. Controversy remains as to whether adenomyomatosis of the gallbladder and porcelain gallbladder are associated
\end{abstract}

Offprint requests to: M. Miyazaki

Received: October 1, 2007 / Accepted: October 22, 2007 with gallbladder cancer. With respect to ampullary carcinoma, adenoma of the ampulla is considered to be a precancerous lesion. This article discusses the risk factors for cancerous and precancerous lesions of the biliary tract and prophylactic treatment for these factors.

Key words Biliary tract neoplasms $\cdot$ Risk factors $\cdot$ Prophylaxis therapy · Gallstones · Pancreaticobiliary maljunction · Precancerous conditions $\cdot$ Gallbladder $\cdot$ Guidelines

\section{Introduction}

One of the possible causes of biliary tract cancer may be chronic and continuous stimulation of the biliary tract, and cholangitis due to gallstones and the reflux of pancreatic juice into the biliary tract. ${ }^{1,2}$ The underlying diseases that potentially cause such a condition include pancreaticobiliary maljunction $(\mathrm{PBM}){ }^{3}$ primary sclerosing cholangitis (PSC), ${ }^{4-7}$ chronic cholecystitis, ${ }^{1}$ gallstones, ${ }^{1,8}$ and adenomyomatosis. ${ }^{9}$ In such patients, chronic inflammation potentially causes pathological changes of the biliary epithelium resulting in precursors of biliary tract cancer. ${ }^{1}$

Although curative resection is the only treatment for biliary tract cancer that achieves long-term survival, patients with advanced cancer have only a limited prognosis even after radical surgical resection. To improve the long-term results, therefore, the early detection of precancerous and cancerous lesions, and subsequent cure seem to be essential.

In this article, we discuss the predisposing factors for bile duct cancer, gallbladder cancer, and ampullary car- 
cinoma, using a format of clinical questions (CQs) and responses. In the responses to the CQs, recommendations for treatment are noted (grades of these recommendations are defined in Table $1^{10}$ ). Also, levels of evidence are given (in parentheses) for findings in reference citations (see definitions of levels in Table $2^{10}$ ).

CQ 1 What are the risk factors for biliary tract and ampullary carcinomas?

Pancreaticobiliary maljunction (PBM) with bile duct dilatation and primary sclerosing cholangitis (PSC) are risk factors for biliary tract cancer (see side memos 1 and 2). PBM, particularly that without bile duct dilatation, is a risk factor for gallbladder cancer.

There are no evident risk factors for ampullary carcinoma.

The prevalence rate of biliary tract cancer varies in different geographical regions. Chile and Japan have the highest rate in the world, followed by East Asia and India (level V). ${ }^{1,2}$ Also, there are age differences in the incidence of biliary tract cancer. ${ }^{1}$ It has been reported that the risk factors for biliary tract cancer are, possibly, chronic and continuous stimulation and inflammation of the biliary tract. ${ }^{1,2}$ Polypoid lesions of the gallbladder and adenomyomatosis have been regarded as a risk factors for gallbladder cancer. In this section, we review the respective risk factors for bile duct cancer, gallbladder cancer, and ampullary carcinoma.

Table 1. Strength of recommendations ${ }^{10}$

A, Strongly recommend performing the clinical action

$\mathrm{B}$, Recommend performing the clinical action

$\mathrm{C} 1$, The clinical action may be considered although there is a lack of high-level scientific evidence for its use. May be useful

$\mathrm{C} 2$, Clinical action not definitively recommended because of insufficient scientific evidence. Evidence insufficient to support or deny usefulness

$\mathrm{D}$, Recommend not performing the clinical action
Risk factors for bile duct cancer

Pancreaticobiliary maljunction (see CQ 2)

A retrospective nationwide survey (1990 to 1999) of PBM in Japan revealed that $10.6 \%$ of PBM patients with bile duct dilatation were complicated by biliary tract cancer, and $33.6 \%$ of these biliary tract cancers were bile duct cancer ${ }^{3}$ (level IV). PBM with bile duct dilatation is considered as a risk factor for bile duct cancer.

\section{Primary sclerosing cholangitis (PSC)}

Patients wit PSC carry an increased risk of bile duct cancer. Five percent to $10 \%$ of PSC patients develop bile duct cancer ${ }^{4-7}$ (level V). Bile duct cancer associated with PSC is often advanced with a poor prognosis. PSC, therefore, should be recognized as a risk factor for bile duct cancer.

Controversy remains as to whether bile duct cancer is related to chronic inflammation due to gallstones or some gene mutations ${ }^{8,11,12}$ (level IV).

\section{Risk factors for gallbladder cancer}

\section{Pancreaticobiliary maljunction (see CQ 2)}

There have been many studies that reported PBM as a risk factor for gallbladder cancer. A nationwide survey (1990 to 1999) of PBM revealed that the prevalence rate of biliary tract cancer was $10.6 \%$ in the group with bile duct dilatation, while the prevalence rate was $37.9 \%$ in the group without bile duct dilatation ${ }^{3}$ (level IV). With respect to the PBM patients who developed biliary tract cancer, the incidence of gallbladder cancer was $64.9 \%$ in those with PBM with bile duct dilatation, whereas the incidence was $93.2 \%$ in those with PBM without bile duct dilatation. Therefore, $\mathrm{PBM}$ is an evident risk factor for gallbladder cancer. Of note, the frequency of gallstones in patients with PBM associated with gallbladder cancer is low. ${ }^{13}$

\section{Gallstones and porcelain gallbladder (see CQ 3)}

It has been well established that gallstones are closely associated with gallbladder cancer ${ }^{1,8}$ (level V). It has also been reported that a stone size of more than $3 \mathrm{~cm}$,

Table 2. Levels of evidence $\mathrm{e}^{10}$

\begin{tabular}{ll}
\hline Level I & Systematic review/meta-analysis \\
Level II & One or more randomized clinical trials \\
Level III & Nonrandomized controlled trials \\
Level IV & Analytic epidemiology (cohort studies and case-control studies) \\
Level V & Descriptive study (case reports and case-series studies) \\
Level VI & $\begin{array}{l}\text { Opinions of expert panels and individual experts not based on } \\
\text { patient's data }\end{array}$ \\
\hline
\end{tabular}


a family history of gallbladder cancer, and the duration of cholelithiasis are potential risk factors for developing gallbladder cancer. ${ }^{1,14-16}$ However, there is no evidence of a direct causal relationship between gallstones and gallbladder cancer. Gracie and Ransohoff ${ }^{17}$ followed-up the subsequent history of 123 patients with asymptomatic gallstones for 10 years or longer, and revealed that there was no case of gallbladder cancer reported among that group.

Controversy remains as to whether patients with "porcelain gallbladder" carry a risk of gallbladder cancer. It has been reported that porcelain gallbladder is often complicated by gallbladder carcinoma ${ }^{18,19}$ (level IV), while another report suggests that porcelain gallbladder is not associated with gallbladder carcinoma ${ }^{20}$ (level IV).

\section{Adenoma of the gallbladder (Figs. 1 and 2; also see $C Q 4$ )}

There is consensus regarding the existence of two models through which malignant transformation is produced: the adenoma-carcinoma sequence and the dysplasia-carcinoma sequence. Intestinal and gastric metaplasias appear to be the pathway through which epithelial dysplasia is produced. ${ }^{21}$ Yamagiwa ${ }^{22}$ examined 110 cases of resected gallbladder carcinoma and found dysplasia adjacent to carcinoma in 46 of the 110 cases, and this change was frequently found in lesions at an early stage and in well-differentiated carcinoma (level V).

Kubota et al. ${ }^{23}$ reported that in patients with polypoid lesions of the gallbladder, the respective diameters of adenomas and cancers were $6.9 \mathrm{~mm}$ (range, 4 to $13 \mathrm{~mm}$ ) and $25.7 \mathrm{~mm}$ (range, 5 to $50 \mathrm{~mm}$ ). In that study, $75 \%$ of the adenomas and $13 \%$ of the cancers had a diameter of less than $10 \mathrm{~mm}$. It has also been reported that when a polypoid lesion of the gallbladder is sessile, has a diameter greater than $10 \mathrm{~mm}$, and /or grows rapidly, it is highly likely to be cancerous. ${ }^{23-25}$ In such cases, surgical resection should be recommended.

\section{Adenomyomatosis (Fig. 3; 4)}

Adenomyomatosis has not been considered to have malignant potential. ${ }^{1}$ Nabatame et al. ${ }^{9}$ studied the relationship between adenomyomatosis and gallbladder cancer by examining 4560 gallbladders (2031 from male patients and 2529 from female patients; age 14 to 94 years) resected for gallbladder cancer, gallstones, or other diseases. In that study, the incidence of gallbladder carcinoma was higher in patients with segmental adenomyomatosis $(22 / 334 ; 6.6 \%)$ than in those without (181/4226; $4.3 \% ; P=0.049)$. This difference was more marked in patients equal to or older than 60 years of age $(P<0.001)$. However, the magnitude of risk for gallbladder cancer in patients with adenomyomatosis has not been clearly established. ${ }^{1,26}$

\section{Risk factors for ampullary carcinoma}

Kimura et al. ${ }^{27}$ histologically investigated the papilla of Vater in 576 autopsy cases of elderly people and revealed that the incidences of group 3 and 4 epithelia in the common channel were significantly higher than those in the intraduodenal portion of the bile duct, pancreatic duct, or duodenal epithelia. They also investigated

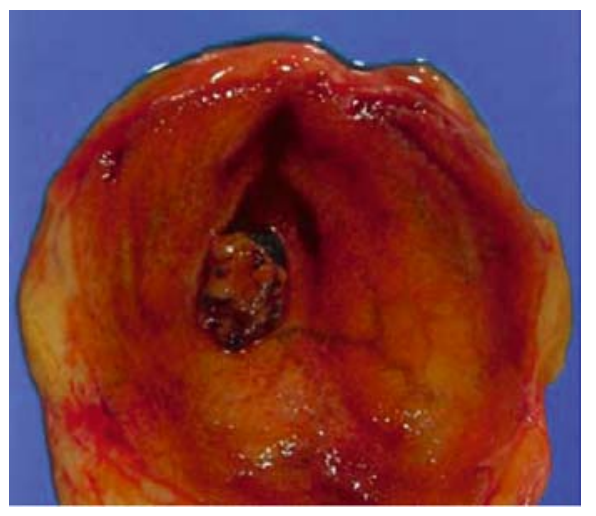

Fig. 1. Macroscopic photograph of adenoma of the gallbladder
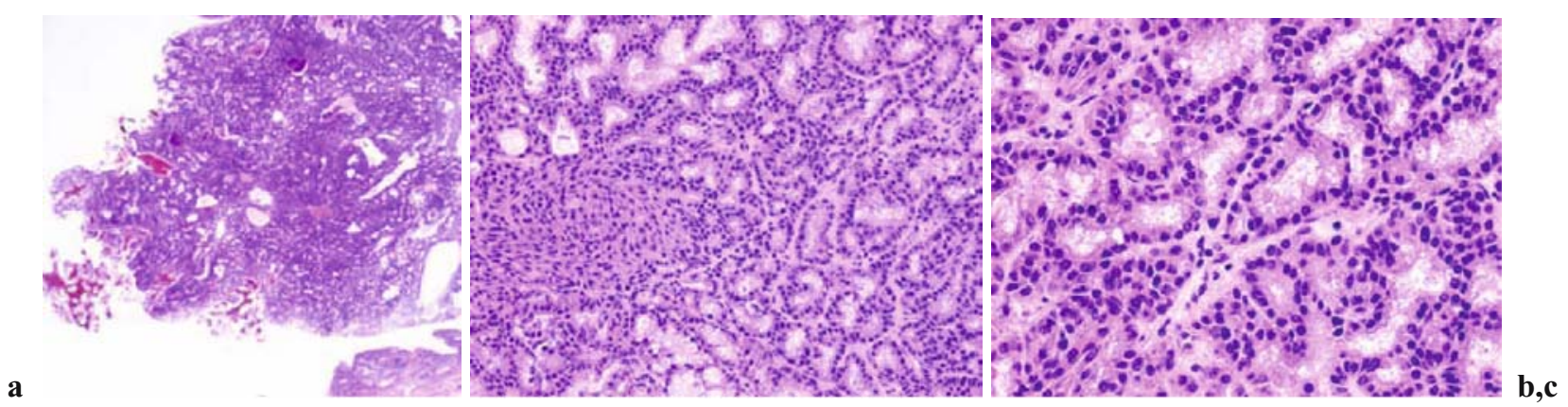

Fig. 2a-c. Histological examination of adenoma of the gallbladder. a Low magnification; b intermediate magnification; c high magnification $(\mathrm{H} \& \mathrm{E})$ 

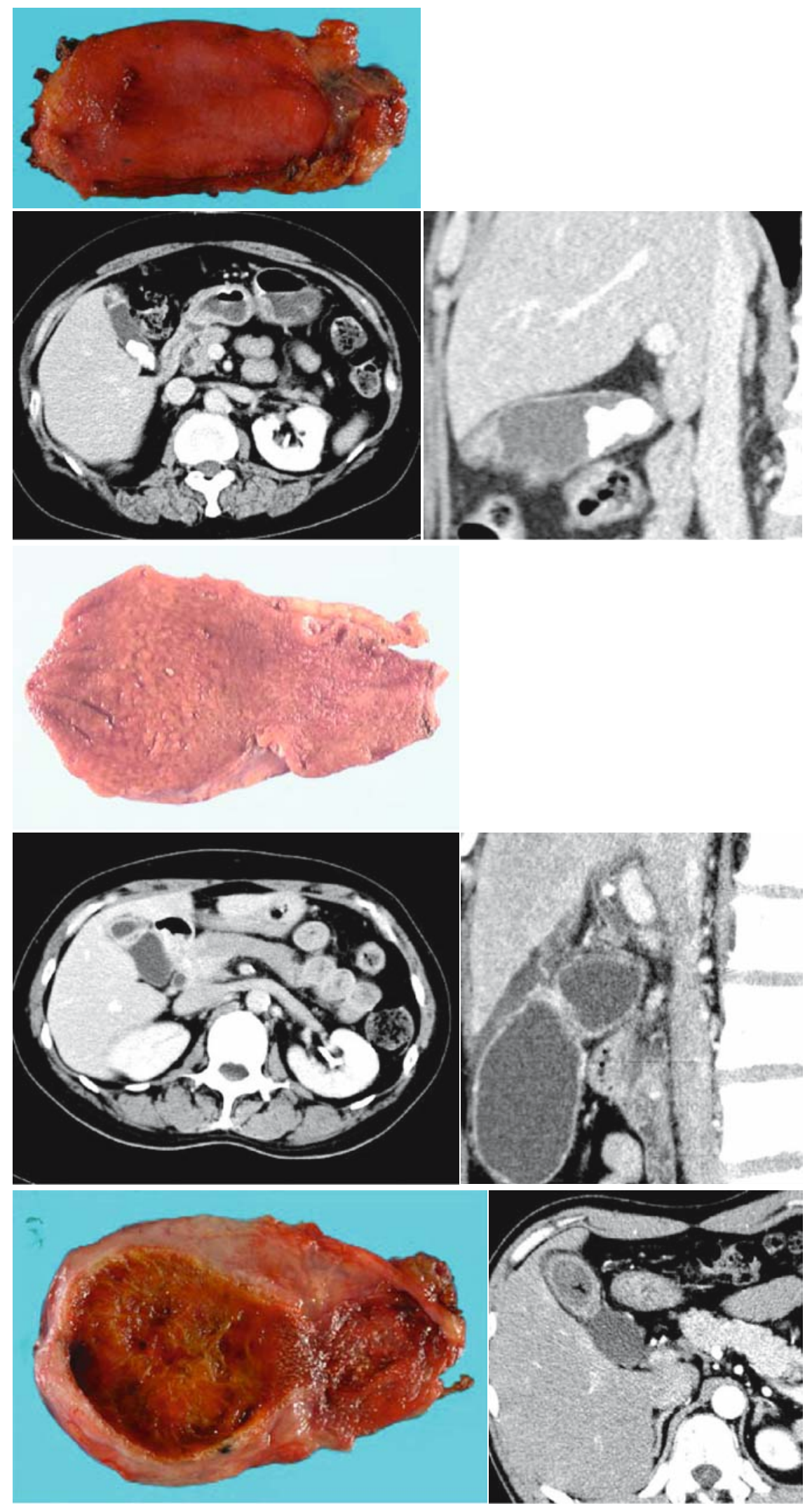

Fig. 3a-c. Findings in adenomyomatosis. a Fundal type: circumscribed hypertrophy of the fundus of the gallbladder with Rokitansky-Aschoff sinus. Case complicated by cholecystolithiasis. Upper, resected specimen; lower left, abdominal computed tomography (CT; cross section); lower right, abdominal CT (sagittal section). b Segmental type: hypertrophy circumscribing the gallbladder with Rokitansky-Aschoff sinus. Upper, resected specimen; lower left, abdominal computed tomography (CT; cross section); lower right, abdominal CT (sagittal section). c Diffuse (segmental-diffuse) type: hypertrophy and Rokitansky-Aschoff sinus from the gallbladder body to the fundus. Gallstones in the fundus. Left, resected specimen; right, abdominal CT 


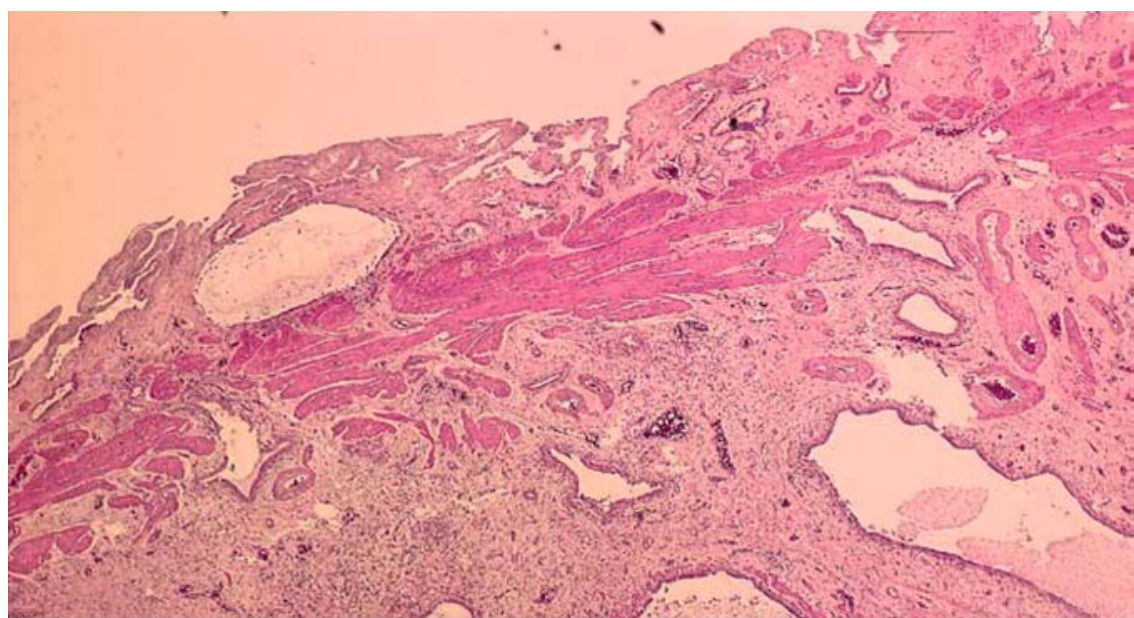

Fig. 4. This Loupe image shows growing and dilating Rokitansky-Aschoff sinuses from the muscularis propria to the subserosa, and the growing smooth muscle fiber and collagen fiber surrounding them (H\&E) resected specimens from the patients with carcinoma of the papilla of Vater and found tha the common channel was the most frequent site for the possible origin of carcinoma (level IV). These results suggest that the common channel is the most important site in the pathogenesis of carcinoma of the papilla of Vater. With regard to the incidence of "adenoma" surrounding carcinoma of the papilla of Vater, the values have been reported to range from $82 \%$ to $91 \%{ }^{27}$ (level IV). Therefore, the adenoma-carcinoma sequence is very important in the pathogenesis of carcinoma of the papilla of Vater. In addition, familial adenomatous polyposis (FAP) is notable for the risk of adenoma in the ampulla of Vater.

\section{Side memo 1}

\section{Primary sclerosing cholangitis (PSC)}

Definition: recurrent or persistent chronic inflammatory disease of extra- and intrahepatic bile ducts resulting in obliterative fibrosis. No effective treatment has been discovered to date, and liver transplant is required in the terminal stage. Although autoimmune abnormality is suspected, the underlying pathogenesis is still unknown.

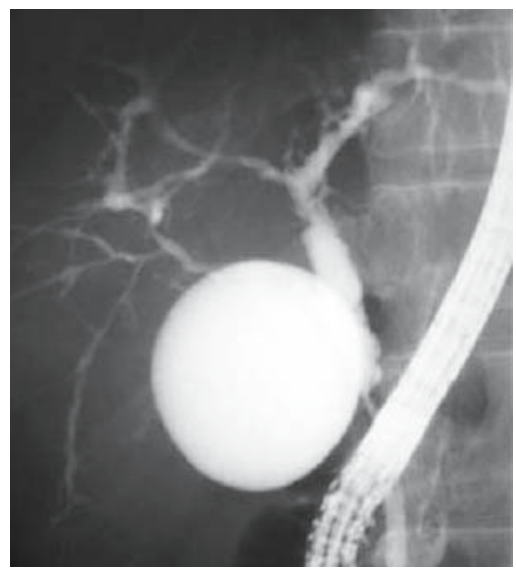

Fig. 5. Endoscopic retrograde cholangiopancreatography image of PSC. Multifocal stricturing of intrahepatic and extrahepatic bile ducts, and shaggy appearance of extrahepatic bile ducts

\section{Diagnostic criteria}

1. Typical radiological findings

Typical radiological findings of bile duct are;

Multifocal stricturing

- Beaded appearance; short or/and annular strictures interspersed among normal or slightly inflamed ducts. This appearance is thought to reflect the fibrous stricture portions and the normal segments of the bile duct. One-fifth of the cases present band-like strictures that are extremely short, and one-fourth present diverticulum-like outpouchings

- Pruned-tree appearance; diminished arborization of the intrahepatic ducts

- Shaggy appearance; diffuse mural irregularities of extrahepatic bile ducts

2. Typical clinical presentation

Important clinical history: inflammatory bowel disease, bile obstruction

Blood examination: high alkaline phosphatase (ALP; two or three times upper limit of normal) for 6 months or more

3. Deductive diagnosis

Exclude secondary sclerotic cholangitis as follows:

- Infectious cholangitis due to AIDS

- Malignant neoplasms or similar disease in the bile duct (excluding PSC accompanied by early-stage cholangiocarcinoma)

- Previous surgery of the biliary tract (excluding cholecystectomy)

- Bacterial cholangitis accompanying biliary tract stricturing or biliary calculus

- Ischemic cholangitis due to floxuridine 
Side memo 2

Diagnostic criteria of pancreaticobiliary maljunction (PBM; see Fig 6)

Definition: PBM is a congenital anomaly consisting of a union of the pancreatic and bile ducts located outside the duodenal wall.

Diagnostic criteria: PBM is diagnosed by either radiological or anatomical findings

1) Radiological findings

It is necessary to confirm the lack of sphincter action at the union of the pancreatic and bile ducts. However, because clarification of the lack of sphincter action is often difficult, the following radiological findings of endoscopic retrograde cholangiopancreatography (ERCP), percutaneous transhepatic cholangiography (PTHC), intraoperative cholangiography, or similar methods are used to verify whether there is a long common channel or a complicated confluence of the pancreatic and bile ducts.

2) Anatomical findings

Confirm the abnormal anatomical confluence of the pancreatic and bile ducts outside the duodenal wall or confirm the complicated confluence by surgery, autopsy, or other procedures. a

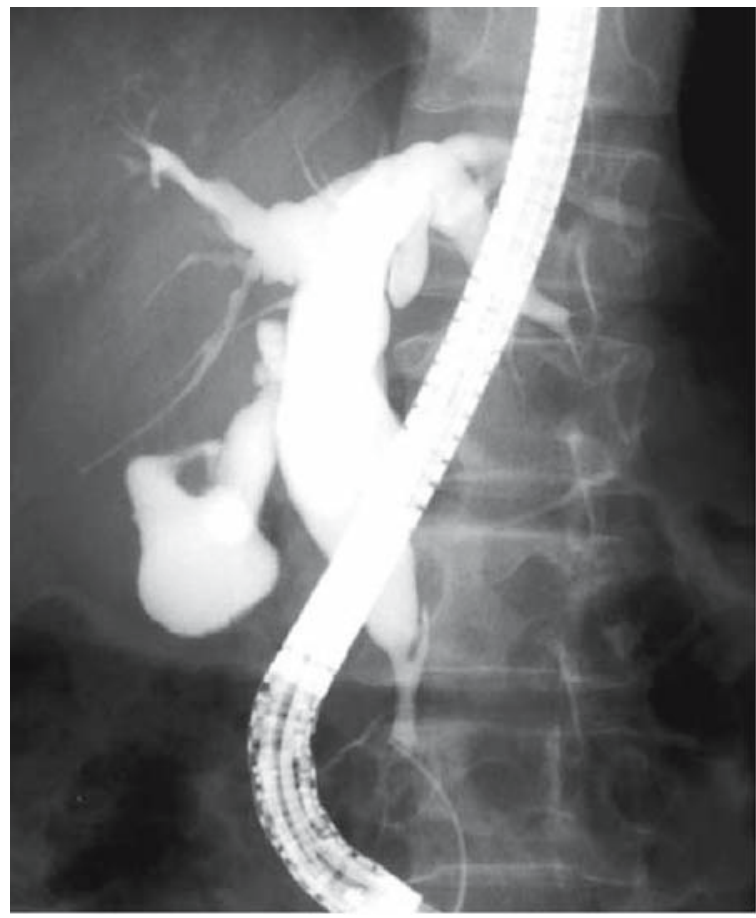

Fig. 6a,b. Endoscopic retrograde cholangiopancreatography (ERCP) image of pancreaticobiliary maljunction (PBM): a patient with congenital bile duct dilatation (Kotani IV a type). a The pancreatic duct joins the biliary duct. b Pancre-

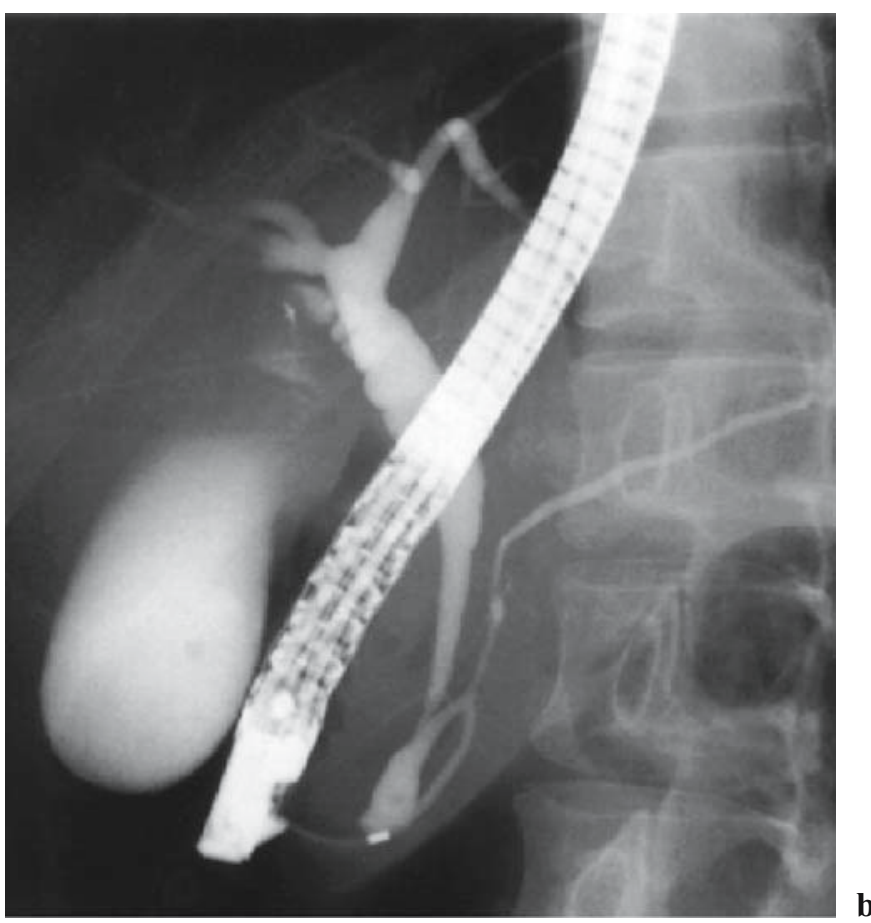

aticobiliary maljunction without bile duct dilatation (Fig 6a, with permission from Koyanagi K, Aoki T, editors. Pancreaticobiliary maljunction. Tokyo: Igaku Tosho Shuppan: 2002. p 25, Fig. $4^{29}$ )
CQ 2 Is prophylactic treatment necessary for pancreaticobiliary maljunction (PBM)?

$P B M$ with bile duct dilatation is a risk factor for bile duct and gallbladder cancer. Prophylactic excision of the gallbladder and common bile duct should be recommended for $P B M$ with bile duct dilatation (recommendation grade $\mathrm{C1}$ ) $P B M$ without bile duct dilatation is a risk factor for gallbladder cancer. A prophylactic cholecystectomy is the appropriate surgical procedure

\section{for PBM without bile duct dilatation. (recommendation grade $B$ )}

As mentioned above, a retrospective nationwide survey (1990 to 1999) of PBM revealed that the incidence of biliary tract cancer was $10.6 \%$ in the group with bile duct dilatation; of these biliary tract cancers, $64.9 \%$ were gallbladder carcinoma and $33.6 \%$ were bile duct cancer. ${ }^{3}$ Therefore, PBM with bile duct dilatation should be considered as a risk factor for bile duct cancer and gallbladder cancer, and in these patients, prophylac- 
tic excision of the gallbladder and extrahepatic bile duct should be considered. Currently, standard prophylactic treatment of PBM with bile duct dilatation consists of cholecystectomy, extrahepatic biliary duct resection, and hepatocholangiojejunostomy. ${ }^{28}$

In the PBM patients without bile duct dilatation, the incidence of biliary tract cancer was $37.9 \%$, and $93.2 \%$ of these patients had gallbladder cancer ${ }^{3}$ (level IV). These reports suggest that PBM without bile duct dilatation should be considered as a risk factor for gallbladder cancer. Therefore, in these patients, excision of the gallbladder should be considered as a minimal prophylactic treatment. Controversy remains as to whether prophylactic bile duct resection is necessary in PBM patients without bile duct dilatation. It has been reported that the long-term results of a prophylactic cholecystectomy without bile duct resection for PBM patients without bile duct dilatation were acceptable ${ }^{29,30}$ (level IV). Also, it has been reported that $K$-ras gene mutation is frequent in the mucosa of the bile duct in PBM patients with bile duct dilatation, while no mutation is observed in PBM patients without bile duct dilatation ${ }^{31}$ (level IV). These reports support a prophylactic cholecystectomy without bile duct resection as being the best treatment option for PBM patients without bile duct dilatation. In contrast, it has been reported that the incidence of bile duct cancer in PBM patients without bile duct dilatation is lower than that in PBM patients with bile duct dilatation, but the incidence seems higher than that in healthy controls ${ }^{3,32}$ (level IV). Moreover, it has been reported that $K$-ras and $p 53$ gene mutations are found frequently even in the nondilated bile duct in patients with $\mathrm{PBM}^{33}$ (level IV). These reports support the possibility that complete excision of the common bile duct may be needed to decrease the risk of bile duct cancer.

CQ 3 Is a cholecystectomy required for asymptomatic cholecystolithiasis?

\section{A cholecystectomy is not advised for asymptomatic cholecystolithiasis (recommendation grade C2)}

It has been well established that gallstones are associated with gallbladder cancer ${ }^{1,8}$ (level V). Not only is there an increased prevalence of gallbladder cancer in patients with gallstones but up to $75 \%$ of patients with gallbladder cancer have been noted to have had gallstones. ${ }^{1}$ It has also been reported that a stone size of more than $3 \mathrm{~cm}$, a family history of gallbladder cancer, and the duration of cholelithiasis are potential risk factors for the development of gallbladder cancer. ${ }^{1,14-16}$ However, there is no evidence of a direct causal rela- tionship between gallstones and gallbladder cancer. Maringhini et al. ${ }^{34}$ followed-up, for more than 31000 person-years a total of 2583 residents of Rochester, Minnesota, who had gallstones. Gallbladder cancer developed in 5 patients after a median follow-up of 13.3 years. The incidence of gallbladder cancer was significantly higher than expected for men but not for women (153 vs 18 per 100000 person-years). However, these authors do not recommend cholecystectomy, because the absolute incidence and the total numbers of men and women who developed gallbladder cancer were low. Moreover, Gracie and Ransohoff ${ }^{17}$ followed up the subsequent history of 123 patients with asymptomatic gallstones for 10 years or longer, and revealed that there was no case of gallbladder cancer reported among that group. This low rate of cancer development in asymptomatic patients with gallstones is similar to that noted in other reports $(0.01 \% / \text { year- } 0.02 \% / \text { year })^{1,35}$ and, in general, this low rate has lent support to the practice of not performing prophylactic cholecystectomy in patients with asymptomatic cholelithiasis.

Controversy remains as to whether patients with calcification of the wall of the gallbladder, referred to as "porcelain gallbladder" carry a risk of gallbladder cancer. It has been reported that porcelain gallbladder is often complicated by gallbladder carcinoma ${ }^{18,19}$ (level IV). However, Towfigh et al. ${ }^{20}$ have reported that porcelain gallbladder is not associated with gallbladder carcinoma(levelIV). The pathogenicmechanism underlying the relationship of calcification to gallbladder cancer also remains unknown.

CQ 4 Is a cholecystectomy necessary for polypoid lesions of the gallbladder (see side memo 3)

When a polypoid lesion of the gallbladder is sessile, has a diameter greater than $10 \mathrm{~mm}$, and/or grows rapidly, it is highly likely to be cancerous and should be resected (recommendation grade B)

Currently, adenoma and dysplasia have been regarded as a precancerous lesions of the gallbladder. ${ }^{21}$ Intestinal and gastric metaplasia seems to be the pathway through which the epithelial dysplasia is produced. ${ }^{21,22}$ Yamagiwa $^{22}$ examined 110 cases of resected gallbladder carcinoma and revealed that dysplasia adjacent to carcinoma was found in 46 of the 110 cases, and this change was frequently found in lesions at the early stage and in well-differentiated carcinoma (level V).

Kubota et al. ${ }^{23}$ evaluated 72 patients who underwent cholecystectomy for polypoid lesions of the gallbladder. In that study, histological examination showed cholesterol polyps in 47 patients, adenoma in 8 , cancers in 16 , 
and an inflammatory polyp in 1 . The respective diameters of the adenomas and cancers were $6.9 \mathrm{~mm}$ (range, 4 to $13 \mathrm{~mm}$ ) and $25.7 \mathrm{~mm}$ (range, 5 to $50 \mathrm{~mm}$ ); $75 \%$ of the adenomas and $13 \%$ of the cancers had a diameter of less than $10 \mathrm{~mm}$ (level IV). Chijiiwa and Tanaka ${ }^{36}$ also examined 44 patients who underwent cholecystectomy for polypoid lesions of the gallbladder, and reported that the sex ratio, symptoms, and the presence of gallstones were not significantly different between patients with carcinoma and those with benign polypoid lesions, and that the size $(>10 \mathrm{~mm})$, number of polypoid lesions ( single), and age ( $>$ or $=60$ years) were significant indicators of carcinoma.

It has been reported that when a polypoid lesion of the gallbladder is sessile, has a diameter greater than $10 \mathrm{~mm}$, and/or grows rapidly, it is highly likely to be cancerous $^{23-25,36-39}$ (level IV). In such cases, surgical resection should be considered.
Side memo 3

Polypoid lesions of the gallbladder (see Figs. 7-10)

Definition: "Polypoid" is the general term for torous lesions protruding into the lumen of the gallbladder, no matter whether they are neoplastic or nonneoplastic. The majority

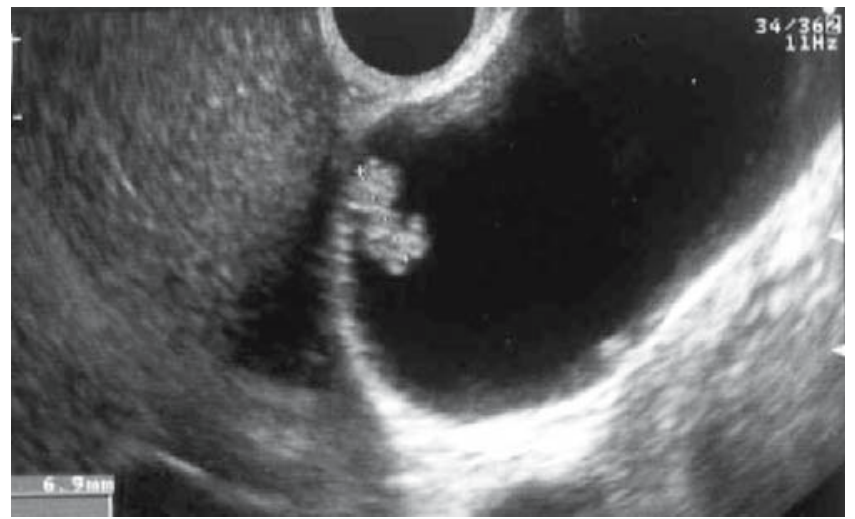

Fig. 7. Ultrasound image of a cholesterol polyp: sessile polyp that has a higher echoic signal than the liver

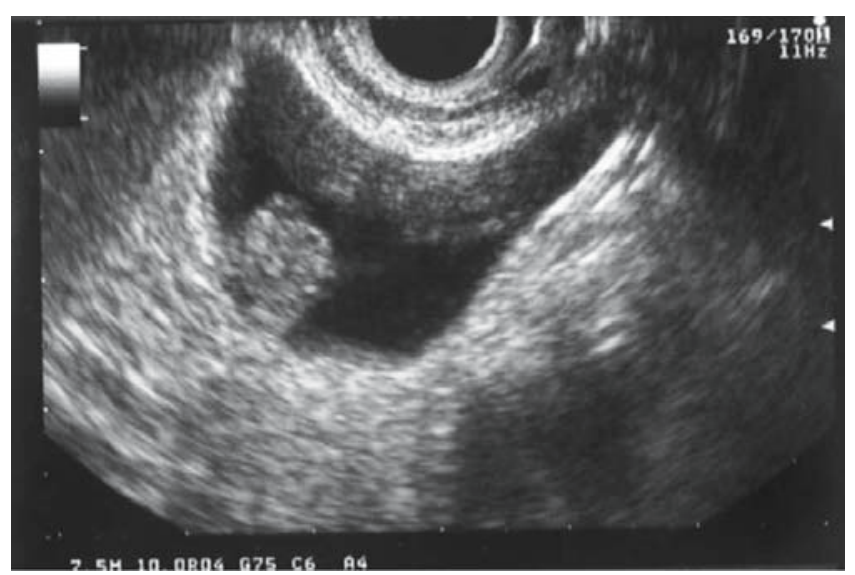

Fig. 8. Ultrasound image of adenoma of the gallbladder: this image indicates a semipedunculate polyp with isoechoic signal of polypoid lesions are nonneoplastic lesions such as cholesterol polyp, adenomyomatosis, or inflammatory polyp. Benign neoplastic lesions include adenoma and metaplastic polyp, while malignant neoplastic lesions include gallbladder cancer

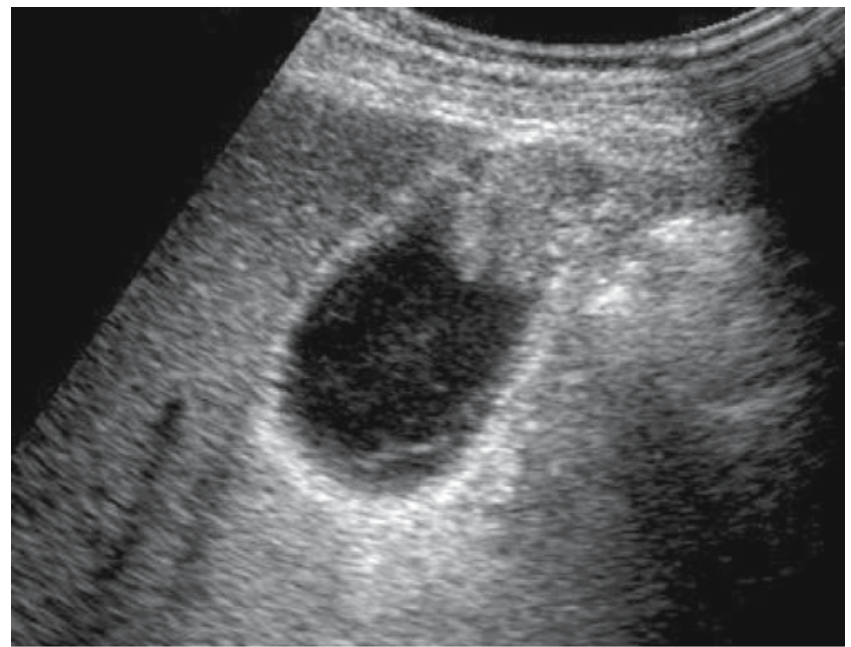

Fig. 9. Ultrasound image of early-stage gallbladder carcinoma: a pedunculated protrusion with a relatively high echoic signal. Depth of tumor invasion is limited to the mucosal layer

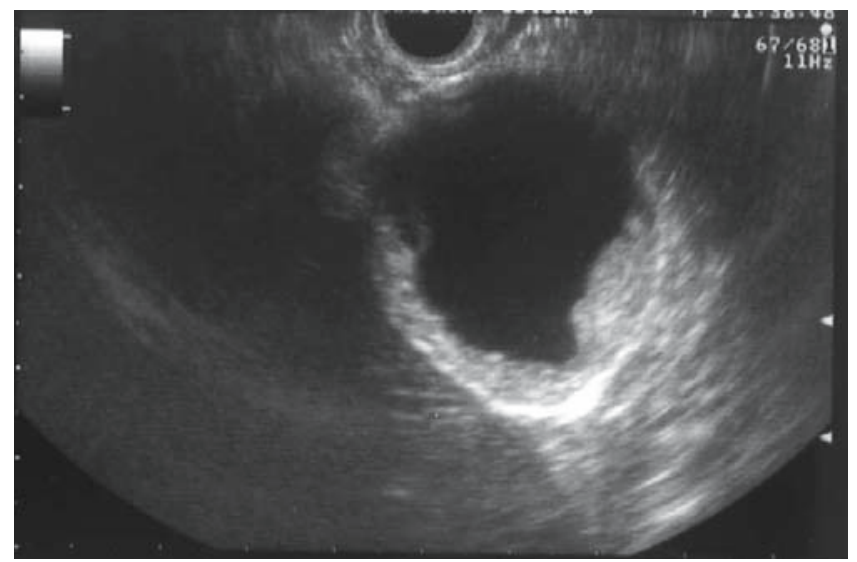

Fig. 10. Ultrasound image of gallbladder carcinoma: sessile protruding lesion. Depth of tumor invasion is to the subserosa 
Acknowledgment. We would like to express our deep gratitude to the members of the the Japanese Association of Biliary Surgery, the Japanese Society of HepatoBiliary-Pancreatic Surgery, and the Japan Society of Clinical Oncology, who provided us with great support and guidance in the preparation of the Guidelines. This process was conducted as part of the Integrated Research Project for Assessing Medical Technology 2005 and 2006 sponsored by the Japanese Ministry of Health, Labour, and Welfare.

We truly appreciate the following active working members who developed the draft of the evidencebased clinical practice Guidelines for the treatment of biliary tract cancer (Japanese version, 2007): Masahiro Kai (Miyazaki), Yasutoshi Kimura (Sapporo), Shigeaki Sawada (Toyama), Hiroaki Shimizu (Chiba), Hisatoshi Nakagawara (Kanazawa), Kohei Nakachi (Kashiwa), and Hiroyuki Yoshitome (Chiba). We also appreciate very much the following members who reviewed and approved the final Japanese version of the guidelines: Hiromitsu Saisyo (Ichikawa), Munemasa Ryu (Chiba), Satoru Shikata (Kyoto), and Yuji Nimura (Nagoya).

\section{References}

1. Sheth S, Bedford A, Chopra S. Primary gallbladder cancer: recognition of risk factors and the role of prophylactic cholecystectomy. Am J Gastroenterol 2000;95:1402-10.

2. Matsukura N, Yokomuro S, Yamada S, Tajiri T, Sundo T, Hadama $\mathrm{T}$, et al. Association between Helicobacter bilis in bile and biliary tract malignancies: $H$. bilis in bile from Japanese and Thai patients with benign and malignant diseases in the biliary tract. Jpn J Cancer Res 2002;93:842-7.

3. Tashiro $\mathrm{S}$, Imaizumi $\mathrm{T}$, Ohkawa $\mathrm{H}$, Okada $\mathrm{A}$, Katoh $\mathrm{T}$, Kawaharada Y, et al. Pancreaticobiliary maljunction: retrospective and nationwide survey in Japan. J Hepatobiliary Pancreat Surg 2003;10:345-51.

4. Rosen CB, Nagorney DM, Wiesner RH, Coffey RJ, LaRusso NF. Cholangiocarcinoma complicating primary sclerosing cholangitis. Ann Surg 1991;213:21-5.

5. Rosen CB, Nagorney DM. Cholangiocarcinoma complicating primary sclerosing cholangitis. Semin Liver Dis 1991;11:26-30.

6. Callea F, Sergi C, Fabbretti G, Brisigotti M, Cozzutto C, Medicina D. Precancerous lesions of the biliary tree. J Surg Oncol Suppl 1993;3:131-3.

7. Franco J, Saeian K. Biliary tract inflammatory disorders. primary sclerosing cholangitis and primary biliary cirrhosis. Curr Gastroenterol Rep 1999;1:95-101.

8. Hsing AW, Bai Y, Andreotti G, Rashid A, Deng J, Chen J, et al. Family history of gallstones and the risk of biliary tract cancer and gallstones: a population-based study in Shanghai, China. Int J Cancer 2007;121:832-8.

9. Nabatame N, Shirai Y, Nishimura A, Yokoyama N, Wakai T, Hatakeyama K. High risk of gallbladder carcinoma in elderly patients with segmental adenomyomatosis of the gallbladder. J Exp Clin Cancer Res 2004;23:593-8.

10. Takada T, Miyazaki M, Miyakawa S, Tsukada K, Nagino M, Kondo $\mathrm{S}$, et al. Purpose, use, and preparation of clinical practice guidelines for the management of biliary tract and ampullary carcinomas. J Hepatobiliary Pancreat Surg 2008;15:2-6.
11. Su WC, Shiesh SC, Liu HS, Chen CY, Chow NH, Lin XZ. Expression of oncogene products HER2/Neu and Ras and fibrosisrelated growth factors bFGF, TGF-beta, and PDGF in bile from biliary malignancies and inflammatory disorders. Dig Dis Sci 2001;46:1387-92.

12. Matsumoto Y, Fujii H, Itakura J, Matsuda M, Yang Y, Nobukawa B, Suda K. Pancreaticobiliary maljunction: pathophysiological and clinical aspects and the impact on biliary carcinogenesis. Langenbecks Arch Surg 2003;388:122-31.

13. Tanaka K, Nishimura A, Yamada K, Ishibe R, Ishizaki N, Yoshimine M, et al. Cancer of the gallbladder associated with anomalous junction of the pancreatobiliary duct system without bile duct dilatation. Br J Surg. 1993;80:622-4.

14. Kumar JR, Tewari M, Rai A, Sinha R, Mohapatra SC, Shukla HS. An objective assessment of demography of gallbladder cancer. J Surg Oncol. 2006;93:610-4.

15. Diehl AK. Gallstone size and the risk of gallbladder cancer. JAMA. 1983;250:2323-6.

16. David FR, William AG. Treatment of gallstones. Ann Intern Med 1993;119:606-19.

17. Gracie WA, Ransohoff DF. The natural history of silent gallstones. N Engl J Med 1982;307:798-800.

18. Stephen AE, Berger DL. Carcinoma in the porcelain gallbladder: a relationship revisited. Surgery 2001;129:699-703.

19. Tazuma S, Kajiyama G. Carcinogenesis of malignant lesions of the gallbladder. The impact of chronic inflammation and gallstones. Langenbecks Arch Surg 2001;386:224-9.

20. Towfigh S, McFadden DW, Cortina GR, Thompson JE, Tompkins $\mathrm{RK}$, Chandler C, et al. Porcelain gallbladder is not associated with gallbladder carcinoma. Am Surg 2001;67:7-10.

21. Roa I, de Aretxabala X, Araya JC, Roa J. Preneoplastic lesions in gallbladder cancer. J Surg Oncol 2006;93:615-23.

22. Yamagiwa H. Mucosal dysplasia of gallbladder: isolated and adjacent lesions to carcinoma. Jpn J Cancer Res 1989;80:23843.

23. Kubota K, Bandai Y, Noie T, Ishizaki Y, Teruya M, Makuuchi M. How should polypoid lesions of the gallbladder be treated in the era of laparoscopic cholecystectomy? Surgery 1995;117:481-7.

24. Ishikawa O, Ohhigashi H, Imaoka S, Nakaizumi A, Kitamura T, Sasaki Y, et al. The difference in malignancy between pedunculated and sessile polypoid lesions of the gallbladder. Am J Gastroenterol 1989;84:1386-90.

25. Sugiyama M, Atomi Y, Yamato T. Endoscopic ultrasonography for differential diagnosis of polypoid gallbladder lesions: analysis in surgical and follow up series. Gut 2000;46:250-4.

26. Aldridge MC, Bismuth $\mathrm{H}$. Gallbladder cancer: the polyp-cancer sequence. Br J Surg 1990;77:363-4.

27. Kimura W, Futakawa N, Zhao B. Neoplastic diseases of the papilla of Vater. J Hepatobiliary Pancreat Surg 2004;11:223-31.

28. Sekido H, Shomada H, Morita T, Matsuo K, Kunihoro O, Miura $\mathrm{Y}$, et al. Indications for surgery and optimal extent of resection of the bile duct: surgical treatment for pancreaticobiliary maljunction. In: Koyanagi K, Aoki T, editors. Pancreaticobiliary maljunction. Tokyo: Igaku Tosho Shuppan; 2002. p. 303-10.

29. Kobayashi S, Asano T, Yamasaki M, Kenmochi T, Saigo K, Ochiai T. Prophylactic excision of the gallbladder and bile duct for patients with pancreaticobiliary maljunction. Arch Surg. 2001;136: 759-63.

30. Ohuchida J, Chijiiwa K, Hiyoshi M, Kobayashi K, Konomi H, Tanaka M. Long-term results of treatment for pancreaticobiliary maljunction without bile duct dilatation Arch Surg. 2006;141: 1066-70.

31. Masuhara S, Kasuya K, Aoki T, Yoshimatsu A, Tsuchida A, Koyanagi Y. Relation between K-ras codon 12 mutation and p53 protein overexpression in gallbladder cancer and biliary ductal epithelia in patients with pancreaticobiliary maljunction. J Hepatobiliary Pancreat Surg 2000;7:198-205.

32. Ando H, Ito T, Nagaya M, Watanabe Y, Seo T, Kaneko K. Pancreaticobiliary maljunction without choledochal cysts in infants and 
children: clinical features and surgical therapy. J Pediatr Surg 1995;30:1658-62.

33. Matsubara T, Zhi LZ, Miura H, Sakurai Y, Imazu H, Imai H, et al. High carcinogenic potential of biliary epithelium in patients with pancreaticobiliary maljunction. In: Koyanagi K, Aoki T, editors. Pancreaticobiliary maljunction. Tokyo: Igaku Tosho Shuppan; 2002. p. 285-94.

34. Maringhini A, Moreau JA, Melton LJ 3rd, Hench VS, Zinsmeister AR, DiMagno EP. Gallstones, gallbladder cancer, and other gastrointestinal malignancies. An epidemiologic study in Rochester, Minnesota. Ann Intern Med 1987;107:30-5.

35. Attili AF, De Santis A, Capri R, Repice AM, Maselli S. The natural history of gallstones: the GREPCO experience. The GREPCO Group. Hepatology 1995;21:655-60.
36. Chijiiwa K, Tanaka M. Polypoid lesion of the gallbladder: indications of carcinoma and outcome after surgery for malignant polypoid lesion. Int Surg 1994;79:106-9.

37. Myers RP, Shaffer EA, Beck PL. Gallbladder polyps: epidemiology, natural history and management. Can J Gastroenterol 2002;16:187-94.

38. Mainprize KS, Gould SWT, Gilbert JM. Surgical management of polypoid lesions of the gallbladder. Br J Surg 2000;87:414-7.

39. Akatsu T, Aiura K, Shimazu M, Ueda M, Wakabayashi G, Tanabe $\mathrm{M}$, et al. Can endoscopic sonography differentiate nonneoplastic from neoplastic gallbladder polyps? Dig Dis Sci 2006;51:41621. 